\title{
Beyond Response: Aiming for Quality Remission in Depression
}

Sidney H. Kennedy

Received: September 15, 2021 / Accepted: December 17, 2021 / Published online: March 5, 2022

(c) The Author(s) 2022

\section{ABSTRACT}

To define treatment response in depression as at least a $50 \%$ reduction in total symptom severity is to accept that up to half of patients will continue to have residual symptoms, most commonly low mood/loss of interest, cognitive problems, lack of energy, and difficulty sleeping. In fact, patients' goals for treatment are to return to premorbid levels of functioning. This highlights the importance of assessing both functional outcomes and symptom improvement when evaluating the efficacy of antidepressant medication. Not all patients who achieve symptomatic response/remission will achieve a functional response/remission. In two studies (one with agomelatine and one with escitalopram), $54 \%$ of patients receiving agomelatine and $47 \%$ of those receiving escitalopram achieved a symptomatic response, and $53 \%$ of patients in each study achieved a functional response. However, $42 \%$ of patients receiving agomelatine and $35 \%$ of those receiving escitalopram had both a symptomatic

S. H. Kennedy $(\bowtie)$

Centre for Depression and Suicide Studies, St

Michael's Hospital, University of Toronto, 193

Yonge Street, Suite 6-001A, Toronto, ON M5B 1M4,

Canada

e-mail: sidney.kennedy@uhn.ca and a functional response. The four symptoms of depression with the most marked effect on function are sad mood, impaired concentration, fatigue, and loss of interest. Low energy is particularly associated with poor occupational functioning, highlighting the importance of ongoing assessment of patients with depression, focusing particular attention on the symptoms that affect their ability to function, such as fatigue. Depending on the type of residual symptoms, some patients may benefit from combination therapy, such as adding dopamine modulator therapy. Antidepressant therapy is only effective if patients continue to take their medication, and high rates of early discontinuation have been reported. Therefore, when selecting treatment for depression, physicians can maximize the likelihood of adherence and persistence by taking into account both the antidepressant efficacy of treatment, its adverse effects and acceptability to patients.

Keywords: Depression;

Treatment; Antidepressants; Residual symptoms; Functioning; Response; Adherence; Persistence 
Key Summary Points

Symptomatic improvement is an early sign of antidepressant treatment response but functional outcomes provide an indicator of meaningful change.

Symptomatic and functional outcomes do not always overlap, as patients may achieve a symptomatic response without achieving a functional response, and vice versa.

The symptoms with the greatest impact on function are sad mood, impaired concentration, fatigue, and loss of interest.

Patients should be monitored for residual symptoms, and treatment tailored towards improvement in both symptoms and function.

\section{INTRODUCTION}

For the last 30 years, the accepted outcome to confirm the efficacy of antidepressant therapy in clinical trials has been 'response', i.e., at least a 50\% reduction in total symptom severity [1], although the merits of this dichotomy have been challenged [2]. Under this definition, many patients continue to have residual symptoms, and are therefore at high risk of developing recurrent or chronic depression, and suicidality [1]. While measurement-based care, particularly using self-reported measures, such as the Patient Health Questionnaire-9 (PHQ-9) [3] or Quick Inventory of Depressive SymptomsSelf Report (QIDS-SR) [4], is gaining acceptance in clinical practice, these instruments continue to measure only symptomatic outcomes, which do not necessarily correlate with functional outcomes. This emphasizes the importance of capturing both symptomatic and functional data when assessing antidepressant outcome.

The purpose of this commentary is to describe, with specific reference to agomelatine, the measurement of functional outcomes in depression, and to highlight the importance of functional outcome assessment as part of an integrated approach to defining recovery, utilizing data from two clinical studies assessing agomelatine [5] and escitalopram [6].

\section{IMPORTANCE OF FUNCTIONAL OUTCOMES}

A systematic review on the relationship between symptoms and function in patients with depression by McKnight and colleagues identified symptom change as an early sign of treatment response, while functional outcomes acted as indicators of a meaningful change [7]. The importance of functional outcome assessment in depression was highlighted by the Canadian Network for Mood and Anxiety Treatments consensus recommendations, published in 2015 (Table 1) [8]. These recommendations include the need for measurementbased care using valid and reliable tools to evaluate both symptoms and function. Examples of these tools include the Sheehan Disability Scale (SDS; Fig. 1) [9]. The SDS consists of a set of questions asking patients to grade the extent to which their symptoms have disrupted three aspects of their lives, work/school, social life, and family life/home responsibilities, on a scale from 0 (not at all) to 10 (extremely) [9]. The SDS is a simple tool that can be quickly used to assess the impact of depression in patient's functioning during routine clinical practice. Another potential tool is the Depression Inventory Development scale, which rates various symptoms including cognition, anhedonia, and fatigue (Fig. 1). In this scale, patients are asked to rate the frequency and intensity of the impact of depression on executive function, concentration, memory, social activities, sexual activity, hobbies and pastimes, drive/motivation, daytime sleepiness, emotional fatigue, and physical weakness [10]. 
Table 1 Consensus recommendations on functional outcomes in depression from the Canadian Network for Mood and Anxiety Treatments [8]

All stakeholders should recognize the clinical significance of functional outcomes in the management of major depressive disorder

Valid and reliable tools for measuring functional outcomes should be developed, evaluated, and disseminated

Clinical trials should be designed with functional outcomes as primary or co-primary outcomes

Stakeholders involved in funding, regulation, and knowledge transition of clinical trials should promote and ensure the inclusion of functional outcomes

Measurement-based care should incorporate functional outcome measures

Research about functional outcomes should be shared widely through integrated knowledge translation strategies

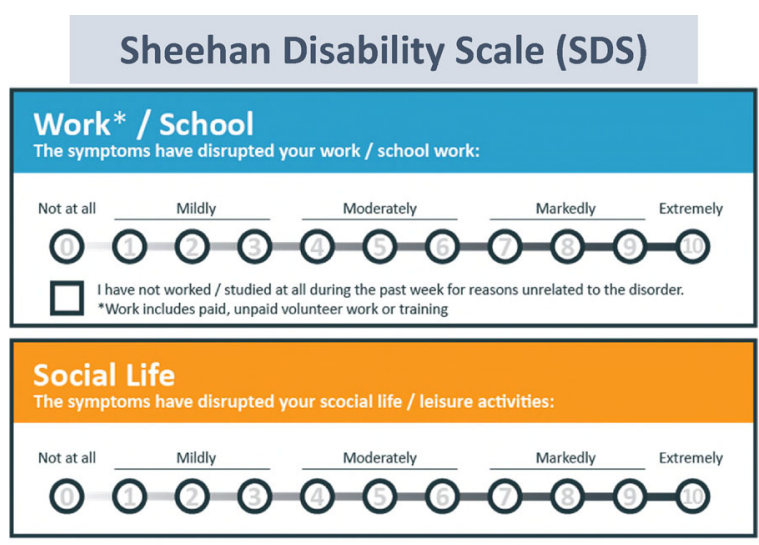

Family Life / Home Responsibilities

The symptoms have disrupted your family life / home responsibilities:

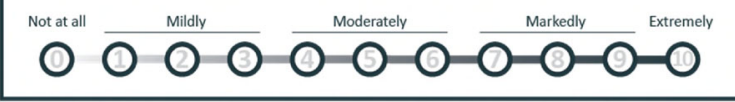

Fig. 1 The Sheehan Disability Score [9]. Reprinted with permission from Handbook of Psychiatric Measures (Copyright (C) 1983), American Psychiatric Association. All rights reserved

\section{IMPACT OF TREATMENT ON FUNCTION}

To illustrate the impact of treatment on patient functioning, agomelatine was selected because there are recent data on the effects of agomelatine on this outcome in patients with major depressive disorder (MDD), using SDS to rate function $[5,11]$. Three doses of agomelatine
(10 mg/day, $25 \mathrm{mg} /$ day, or a starting dose $25 \mathrm{mg} /$ day titrated to $50 \mathrm{mg} /$ day) were compared with placebo over 6 months in patients with MDD. The primary endpoint was the change in Hamilton Depression Rating Scale [HDRS (or HAM-D)] total score, while the SDS was assessed at weeks 6 and 24 as a secondary endpoint, with functional remission defined as an SDS score $\leq 6$ [11]. All agomelatine doses significantly reduced HDRS total score compared with placebo $(P<0.0001)$. In addition, at week 24, significantly more patients in the groups receiving agomelatine $25-50 \mathrm{mg}$ /day $(64.1 \%)$ or $25 \mathrm{mg} /$ day group $(52.5 \%)$ than in the placebo group (26.9\%) had achieved functional remission $(P<0.001$ vs. placebo for both dose groups; Fig. 2). This study also demonstrated that a dose of agomelatine of $\geq 25 \mathrm{mg} /$ day is required for a patient to experience a meaningful functional remission [11].

It should be noted that not all patients who achieve a symptomatic response or remission will achieve a functional response or remission. Two studies-a pooled analysis of two randomized placebo-controlled trials of agomelatine (total $n=633$ ) [5] and a single-arm, openlabel, practice-based study of escitalopram $(n=211)$ [6] -investigated both functional and symptomatic response rates with antidepressant pharmacotherapy. In both studies, functional response was defined as an SDS total score $\leq 12$, while symptomatic response was defined as $\geq 50 \%$ reduction in HDRS total score in the agomelatine study, and as $\geq 50 \%$ reduction in 


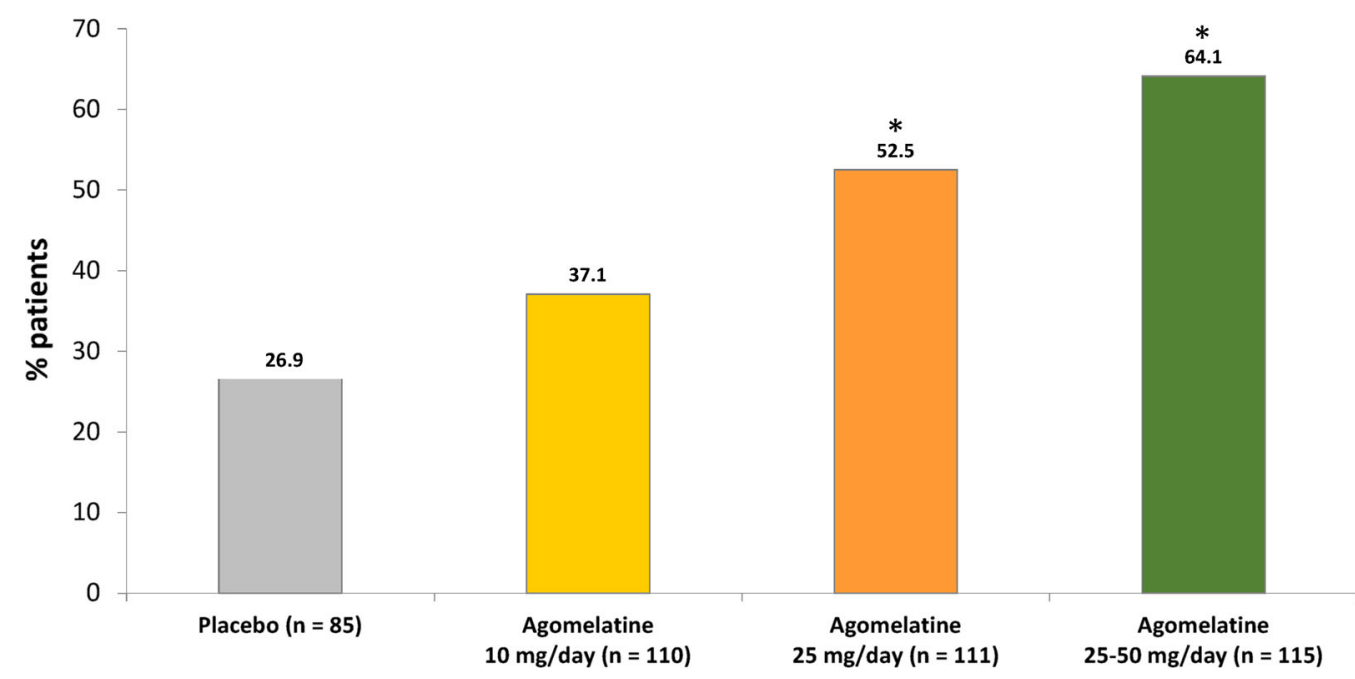

Fig. 2 Effect of agomelatine on the achievement of functional remission (total Sheehan Disability Score of $\leq 2$ ) after 6 months of treatment in a randomized, placebo-controlled study [11]. ${ }^{*} P<0.0001$ vs. placebo

\section{Agomelatine 25-50mg}

Kennedy et al. 2018

\section{Escitalopram 10-20mg}

Kennedy et al. 2019
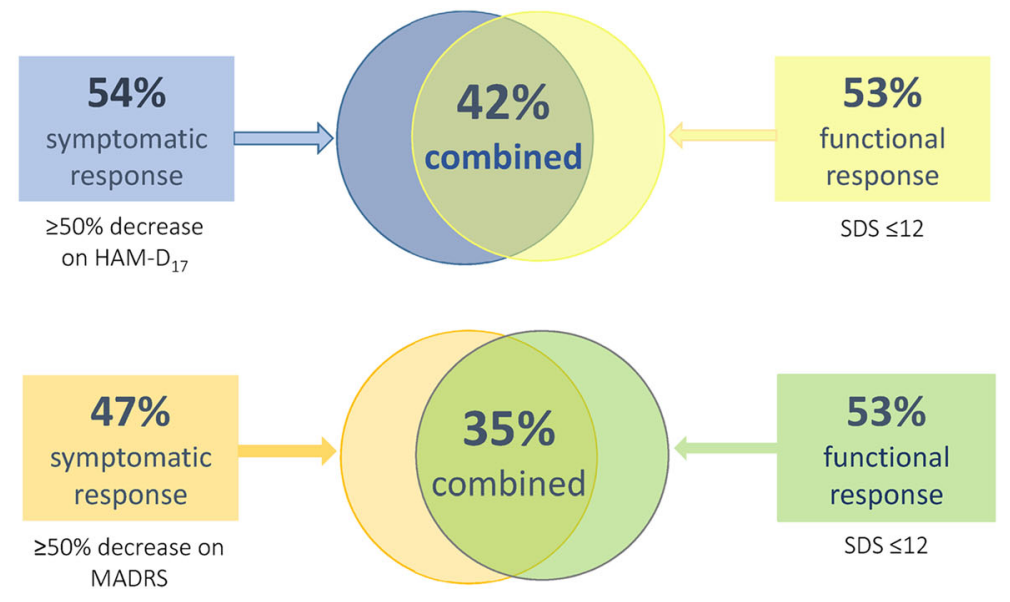

Fig. 3 Rates of symptomatic response, functional response and both, in studies with agomelatine or escitalopram $[5,6]$. HAM-D $D_{17}$ Hamilton Depression Rating Scale,

Montgomery-Åsberg Depression Rating Scale (MADRS) in the escitalopram study $[5,6]$. Approximately $54 \%$ of the patients receiving agomelatine and $47 \%$ of those receiving escitalopram achieved a symptomatic response, and $53 \%$ of patients in each study achieved a functional response. However, the proportion of patients who achieved both a symptomatic and functional response was lower: $42 \%$ in the study with agomelatine [5] and 35\% in the study with
MADRS Montgomery-Åsberg Depression Rating Scale, SDS Sheehan Disability Score

escitalopram (Fig. 3) [6]. The escitalopram study also investigated the rate of symptomatic and functional remission, defined as a MADRS score $\leq 10 \%$ and SDS score $\leq 6$, at 8 and 16 weeks [6]. The rate of remission significantly increased between week 8 and week 16, from $31 \%$ (symptomatic remission) at week 8 to $80 \%$ at week 16, and from $24 \%$ (functional remission) at week 8 to $57 \%$ at week $16(P<0.001)$ [6]. However, the proportion of patients who 
achieved both symptomatic and functional remission was lower at both time-points: $18 \%$ at week 8 and 52\% at week 16 [6]. These data highlight the importance of assessing functional outcomes, because we cannot assume that patients who experience a symptomatic improvement are also achieving a functional improvement.

\section{THE DISCONNECT BETWEEN SYMPTOMS AND FUNCTION}

The differential effect of treatment on symptoms and function is because of the many potential combinations of symptoms that are possible among patients with depression. In an analysis of the Sequenced Treatment Alternatives to Relieve Depression study, four specific symptoms had the greatest impact on function [12]. Similarly, in an earlier naturalistic study in 573 patients receiving pharmacotherapy for depression, $91 \%$ of individuals reported low energy, and this was more strongly correlated with work and social function impairment than other depressive symptoms [13]. Moreover, increased energy was more predictive of an improvement in occupational functioning than was a decrease in the number of depressive symptoms [13]. Furthermore, a prospective study by Conradi and colleagues found that, after 3 years, patients who had achieved remission still experienced residual symptoms of depression, such as low mood/loss of interest, cognitive problems, lack of energy, and difficulty sleeping [14].

These data highlight the importance of ongoing assessment of depressed patients, paying particular attention to the symptoms that affect their ability to function, such as fatigue. They are also a pertinent reminder of the widely cited study by Zimmerman and colleagues in which patients ranked the absence of depressive symptoms sixth on their list of desired outcomes from treatment [15]. Therefore, physicians need to recognize the full range of outcomes that patients want from their treatment (positive mental outlook, emotional control, energy, normal functioning), and incorporate these into their treatment plan and goals in addition to aiming for a change in HDRS score. These data also highlight the importance of using validated patient-reported outcome (PRO) measures to assess response in clinical trials and clinical practice.

One such PRO measure is the Multidimensional Assessment of Thymic States (MAThyS), a 20-item instrument in which patients rate dimensions on a visual analogue scale from 0 to 10 , where 0 represents the most inhibited/inactive end of the spectrum, 10 the most active/excited end, and 5 indicates an average level. An observational study in 1565 outpatients with MDD, who were prescribed agomelatine, found that motivation was the most impaired MAThyS domain at baseline [16]. In addition, the motivation domain (a proxy measure of anhedonia or reward function) was the most sensitive to change, showing the greatest improvement after 2 weeks of treatment, and an early improvement in motivation the best predictor of a symptomatic response at week 16 [16].

\section{COMBINATION THERAPY}

Patients who do not meet the criteria for response during treatment with a selective serotonin reuptake inhibitor (SSRI) may benefit from adjunctive therapy with a dopamine modulator. For example, in the first study of the Canadian Biomarker Integration Network in Depression (CAN-BIND-1), patients who did not meet the response criteria after 8 weeks of treatment with escitalopram (at least 50\% decrease from baseline on the HDRS total score) received adjunctive aripiprazole from week 8 onwards; after 8 weeks of combination therapy, $61 \%$ of these patients achieved symptomatic response and $53 \%$ achieved functional response [6].

Another analysis from the CAN-BIND-1 study showed that patients with high baseline interest-activity scores may benefit from combination therapy with an SSRI and a dopamine agonist [17]. In that analysis, greater loss of interest and reduction in activity scores predicted a poor outcome on escitalopram monotherapy, whereas the same measure was 


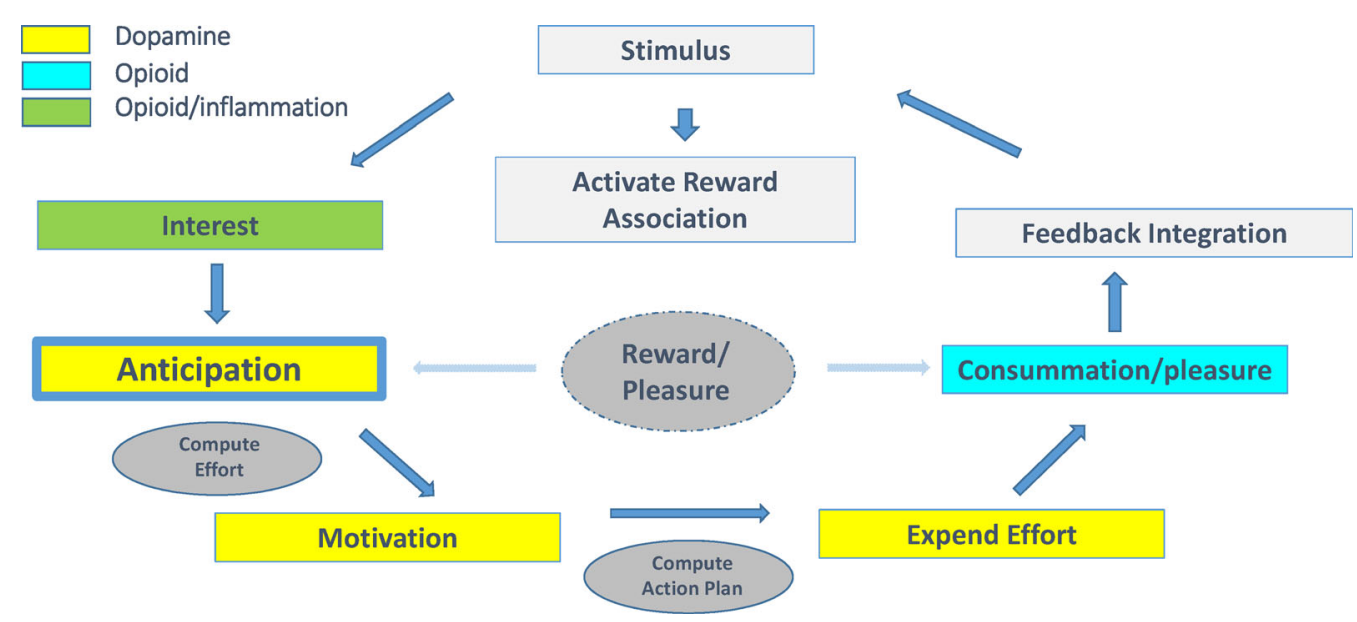

Fig. 4 Model of reward processing [21]. Modified from Rizvi et al. 2016 [21] and Kring and Barch 2014 [22]. Reprinted from Rizvi SJ, et al. Assessing anhedonia in depression: potentials and pitfalls. Neurosci Biobehav Rev. 2016;65:21-35; and Kring E and Barch DM. The

predictive of response improvement when aripiprazole was added [17].

The interest-activity score is a single score derived from six parameters from depression rating scales [18], and reflects anhedonia. Specifically, in the CAN-BIND-1 study, these parameters were 'concentration', 'lassitude', and 'inability to feel' from the MADRS, and 'concentration', 'energy', and 'interest' from the QIDS-SR [17]. The relationship between interest-activity scores and poor response to SSRIs has been reported in other studies [18], suggesting that this parameter may help to predict which patients may benefit from combination therapy.

\section{ANHEDONIA AND RESPONSE PREDICTION}

The concept of 'anhedonia' has evolved since it was first coined by Ribot in 1897 to describe the inability to feel pleasure [19]. Today, anhedonia is understood to include the inability to pursue reward or experience rewarding activities [20]. Several different neurotransmitters are implicated in the stimulus-reward cycle, controlling interest, anticipation, motivation, and action (Fig. 4) [21, 22]. This implies that aspects of motivation and pleasure dimension of negative symptoms: neural substrates and behavioral outputs. Eur Neuropsychopharmacol. 2014;24:725-736, with permission from Elsevier; permission conveyed through Copyright Clearance Center, Inc

anhedonia, such as reward anticipation, may influence the response to antidepressants.

In the CAN-BIND-1 study, participants were asked to complete a Monetary Incentive Delay task, which measures anticipatory and consummatory responses to reward during functional magnetic resonance imaging (fMRI), at baseline and after 2 weeks of treatment with escitalopram [23]. Greater anhedonia severity at baseline was associated with a poor response to escitalopram. Moreover, an early increase in functional connectivity between the ventral striatum and rostral anterior cingulate nucleus on fMRI between baseline and week 2 correlated with the reduction in MADRS score at week 8 [23]. These data suggest that we may soon be able to apply precision psychiatry by identifying patients who are likely to have a remission during antidepressant therapy, by tailoring treatment more effectively.

\section{TREATMENT PERSISTENCE}

Antidepressant therapy is only effective if patients continue to take their medication. Real-world data from the United States have shown that about $50 \%$ of patients discontinued their antidepressant therapy after 3-4 months 
of treatment, and that more than $75 \%$ discontinued treatment within a year [24]. Treatmentrelated adverse effects, particularly those that may interfere with daily function, can have a major impact on patient adherence. The adverse effects of medication perceived by patients to have the greatest effect on their ability to function at work were daytime sleepiness, difficulty sleeping at night, headache, and anxiety/agitation [25]. Therefore, when selecting treatment for depression, physicians need to consider both antidepressant efficacy and acceptability to patients including their ability to work with minimum interference from side effects. In a large-scale metaanalysis of antidepressant treatments conducted by Cipriani and colleagues, agomelatine, escitalopram, and vortioxetine offered the best balance of efficacy and acceptability [26]. Given the relatively high rates of non-response and non-remission with antidepressant monotherapy, augmentation with atypical dopamine agonist antipsychotic agents is a common strategy to achieve better outcomes [27, 28]. However, given the potential for dopamineassociated akathisia and tardive dyskinesia, it is important to carefully monitor the use of these drugs $[29,30]$.

\section{COST-BENEFIT OF EFFECTIVE TREATMENT FOR DEPRESSION}

Because depression has a marked impact on patients' productivity and work performance, effective treatment of depression has the potential to make a significant contribution not only to individual patients' well-being but also to global economic well-being. According to one estimate, each dollar invested in effective treatment of depression and anxiety yields between US\$3 and \$6 in total economic gains, with improved occupational productivity contributing about half of these benefits [31].

\section{CONCLUSION}

Functional remission is an important treatment goal because it is better than symptomatic remission as an indicator of overall recovery. The best outcomes of depression are achieved when the treatment provides an optimal balance between efficacy and tolerability. In the future, it is likely that physicians are to individualize the treatment of depression based on clinical symptoms and bio-signatures [32].

\section{ACKNOWLEDGEMENTS}

The author thanks Ms. Amanda Ceniti, who assisted with preparation of the original presentation and contributed to revision of the manuscript drafts.

Funding. This article is based on a presentation given by Prof. Kennedy at a satellite symposium entitled Road to Recovery for Depressed Patients: From Treatment Initiation to Continuity. The symposium formed part of the programme of the WPA World Congress of Psychiatry, held 10-13 March 2021. The satellite symposium was sponsored by Servier, which also provided funding for medical writing assistance. This supplement has been sponsored by Servier.

Medical Writing. The author thanks Catherine Rees of Springer Healthcare Communications, who prepared the first draft of the manuscript based on the corresponding presentation at WPA World Congress of Psychiatry 2021. Funding for the writing was provided by Servier.

Authorship. The named author of this article meets the International Committee of Medical Journal Editors (ICMJE) criteria for authorship for this article, takes responsibility for the integrity of the work as a whole, and has given their approval for this version to be published.

Author Contributions. Prof. Kennedy prepared the content of the presentation on which this article was based, and reviewed and revised all drafts of the manuscript. 
Prior Presentation. This article is based on a presentation given by the author at a satellite symposium entitled Road to Recovery for Depressed Patients: From Treatment Initiation to Continuity. The symposium formed part of the programme of the WPA World Congress of Psychiatry, held virtually from 10-13 March 2021. The content of the presentation and this article are based on work that has been previously published.

Disclosures. Prof. Kennedy has acted in an advisory role for Allergan, Alkermes, Janssen, Lundbeck, Lundbeck Institute, Otsuka, Pfizer, Servier and Sunovion; has been a speaker for Allergan, Lundbeck, Otsuka, Servier, Sun and Sunovion; has received research grants from the Ontario Brain Institute, Ontario Research Foundation, CIHR, Brain Canada, Janssen, Lundbeck, Otsuka, Pfizer and Servier; and has been a clinical trial investigator in studies sponsored by Janssen, Lundbeck, Pfizer, Abbott and SPOR. He holds stock in Field Trip Health. Servier has participated in the development of this article.

Compliance with Ethics Guidelines. This article is based on previously conducted studies and does not contain any new studies with human participants or animals performed by any of the authors.

Open Access. This article is licensed under a Creative Commons Attribution-NonCommercial 4.0 International License, which permits any non-commercial use, sharing, adaptation, distribution and reproduction in any medium or format, as long as you give appropriate credit to the original author(s) and the source, provide a link to the Creative Commons licence, and indicate if changes were made. The images or other third party material in this article are included in the article's Creative Commons licence, unless indicated otherwise in a credit line to the material. If material is not included in the article's Creative Commons licence and your intended use is not permitted by statutory regulation or exceeds the permitted use, you will need to obtain permission directly from the copyright holder. To view a copy of this licence, visit http://creativecommons.org/licenses/by$\mathrm{nc} / 4.0 /$.

\section{REFERENCES}

1. Culpepper L, Muskin PR, Stahl SM. Major depressive disorder: understanding the significance of residual symptoms and balancing efficacy with tolerability. Am J Med. 2015;128(9 Suppl):S1-15.

2. Hieronymus F, Jauhar S, Østergaard SD, Young AH. One (effect) size does not fit at all: interpreting clinical significance and effect sizes in depression treatment trials. J Psychopharmacol. 2020;34(10): 1074-8.

3. Kroenke K, Spitzer RL, Williams JB. The PHQ-9: validity of a brief depression severity measure. J Gen Intern Med. 2001;16(9):606-13.

4. Rush AJ, Trivedi MH, Ibrahim HM, et al. The 16-item quick inventory of depressive symptomatology (QIDS), clinician rating (QIDS-C), and selfreport (QIDS-SR): a psychometric evaluation in patients with chronic major depression. Biol Psychiatry. 2003;54(5):573-83.

5. Kennedy SH, Heun R, Avedisova A, et al. Effect of agomelatine $25-50 \mathrm{mg}$ on functional outcomes in patients with major depressive disorder. J Affect Disord. 2018;238:122-8.

6. Kennedy SH, Lam RW, Rotzinger S, et al. Symptomatic and functional outcomes and early prediction of response to escitalopram monotherapy and sequential adjunctive aripiprazole therapy in patients with major depressive disorder: A CAN-BIND-1 report. J Clin Psychiatry. 2019;80(2):18m12202.

7. McKnight PE, Kashdan TB. The importance of functional impairment to mental health outcomes: a case for reassessing our goals in depression treatment research. Clin Psychol Rev. 2009;29(3):243-59.

8. Lam RW, Parikh SV, Michalak EE, Dewa CS, Kennedy SH. Canadian network for mood and anxiety treatments (CANMAT) consensus recommendations for functional outcomes in major depressive disorder. Ann Clin Psychiatry. 2015;27(2):142-9.

9. Sheehan DV, Harnett-Sheehan K, Raj BA. The measurement of disability. Int Clin Psychopharmacol. 1996;11(Suppl 3):89-95.

10. Vaccarino AL, Evans $\mathrm{KR}$, Kalali $\mathrm{AH}$, et al. The depression inventory development workgroup: a collaborative, empirically driven initiative to develop a new assessment tool for major depressive 
disorder. Innov Clin Neurosci. 2016;13(9-10): 20-31.

11. Kennedy SH, Avedisova A, Belaidi C, Picarel-Blanchot F, de Bodinat C. Sustained efficacy of agomelatine $10 \mathrm{mg}, 25 \mathrm{mg}$, and $25-50 \mathrm{mg}$ on depressive symptoms and functional outcomes in patients with major depressive disorder. A placebo-controlled study over 6 months. Eur Neuropsychopharmacol. 2016;26(2):378-89.

12. Fried EI, Nesse RM. The impact of individual depressive symptoms on impairment of psychosocial functioning. PLoS ONE. 2014;9(2):e90311.

13. Swindle R, Kroenke K, Braun L. Energy and improved workplace productivity in depression. In: Farquhar I, Summers K, Sorkin A, editors. Investing in health: the social and economic benefits of health care innovation. Bingley: Emerald; 2001. p. 323-41.

14. Conradi HJ, Ormel J, de Jonge P. Presence of individual (residual) symptoms during depressive episodes and periods of remission: a 3-year prospective study. Psychol Med. 2011;41(6):1165-74.

15. Zimmerman M, McGlinchey JB, Posternak MA, Friedman M, Attiullah N, Boerescu D. How should remission from depression be defined? The depressed patient's perspective. Am J Psychiatry. 2006;163(1):148-50.

16. Gorwood P, Vaiva G, Corruble E, Llorca PM, Bayle FJ, Courtet $\mathrm{P}$. The ability of early changes in motivation to predict later antidepressant treatment response. Neuropsychiatr Dis Treat. 2015;11:2875-82.

17. Uher R, Frey BN, Quilty LC, et al. Symptom dimension of interest-activity indicates need for aripiprazole augmentation of escitalopram in major depressive disorder: a CAN-BIND-1 report. J Clin Psychiatry. 2020. https://doi.org/10.4088/JCP. $20 \mathrm{~m} 13229$.

18. Uher R, Perlis RH, Henigsberg N, et al. Depression symptom dimensions as predictors of antidepressant treatment outcome: replicable evidence for interest-activity symptoms. Psychol Med. 2012;42(5):967-80.

19. Berrios GE, Olivares JM. The anhedonias: a conceptual history. Hist Psychiatry. 1995;6(24 Pt 4): 453-70.

20. Thomsen KR. Measuring anhedonia: impaired ability to pursue, experience, and learn about reward. Front Psychol. 2015;6:1409.

21. Rizvi SJ, Pizzagalli DA, Sproule BA, Kennedy SH. Assessing anhedonia in depression: potentials and pitfalls. Neurosci Biobehav Rev. 2016;65:21-35.
22. Kring AM, Barch DM. The motivation and pleasure dimension of negative symptoms: neural substrates and behavioral outputs. Eur Neuropsychopharmacol. 2014;24(5):725-36.

23. Dunlop K, Rizvi SJ, Kennedy SH, et al. Clinical, behavioral, and neural measures of reward processing correlate with escitalopram response in depression: a Canadian Biomarker Integration Network in Depression (CAN-BIND-1) report. Neuropsychopharmacology. 2020;45(8):1390-7.

24. Milea D, Guelfucci F, Bent-Ennakhil N, Toumi M, Auray JP. Antidepressant monotherapy: a claims database analysis of treatment changes and treatment duration. Clin Ther. 2010;32(12):2057-72.

25. Lam RW, Michalak EE, Bond DJ, Tam EM, Axler A, Yatham LN. Which depressive symptoms and medication side effects are perceived by patients as interfering most with occupational functioning? Depress Res Treat. 2012;2012:630206.

26. Cipriani A, Furukawa TA, Salanti G, et al. Comparative efficacy and acceptability of 21 antidepressant drugs for the acute treatment of adults with major depressive disorder: a systematic review and network meta-analysis. Lancet. 2018;391(10128): 1357-66.

27. Cantù F, Ciappolino V, Enrico P, Moltrasio C, Delvecchio G, Brambilla P. Augmentation with atypical antipsychotics for treatment-resistant depression. J Affect Disord. 2021;280(Pt A):45-53.

28. Kennedy SH, Lam RW, McIntyre RS, et al. Canadian Network for Mood and Anxiety Treatments (CANMAT) 2016 clinical guidelines for the management of adults with major depressive disorder: Section 3. Pharmacological treatments. Can J Psychiatry. 2016;61(9):540-60.

29. Coplan J, Gugger JJ, Tasleem H. Tardive dyskinesia from atypical antipsychotic agents in patients with mood disorders in a clinical setting. J Affect Disord. 2013;150(3):868-71.

30. Kumar R, Sachdev PS. Akathisia and secondgeneration antipsychotic drugs. Curr Opin Psychiatry. 2009;22(3):293-9.

31. Chisholm D, Sweeny K, Sheehan P, et al. Scaling-up treatment of depression and anxiety: a global return on investment analysis. Lancet Psychiatry. 2016;3(5):415-24.

32. Trivedi MH, McGrath PJ, Fava M, et al. Establishing moderators and biosignatures of antidepressant response in clinical care (EMBARC): rationale and design. J Psychiatr Res. 2016;78:11-23. 